\title{
Serious Economic Games: Designing a Simulation Game for an Economic Experiment
}

\author{
Danilo Wanner \\ Visual Communication Institute, The Basel School of Design, HGK FHNW, \\ Basel, Switzerland \\ danilo.wanner@fhnw.ch
}

\begin{abstract}
This project proposes the design of a Serious Game for an economic experiment. The analysis of existing games and research into current practices and requirements in economic experimentation are combined to create a Serious Economic Game. It is found that laboratory experiments are conducted in a way that prevents immersion. A game using a visual representation of the economic model and presenting input in the form of gameplay decisions has the potential to provoke a naturalistic player response through immersion. Case studies of existing games show various ways in which to achieve player engagement and immersion. The employed ,aesthetic“ approach to game design involves detailed, photorealistic graphics and sound bound to diegesis to demand the attention of players and therefore facilitate immersion.
\end{abstract}

Keywords: Serious Games, Economic Experiment, Design Research, Digital Aesthetics, Game Aesthetics, Aesthetic Approach, Game Design.

\section{Introduction}

Games have the remarkable power to capture and immerse their players. In recent years, a field called ,serious games“ has gained significant interest, inspired by the huge popularity and success of games in the entertainment industry. Serious Games try to tap the motivational power of games to help achieve real-world goals and solve real problems. While most Serious Games are used in the field of learning, this thesis adapts game capabilities for an economic experiment. It proposes a Serious Economic Game, played by Brazilian farmers, to evaluate their economic decisions under different conditions. The game was put to use by a research project of the ETH Zurich, which investigates one of the largest environmental issues on the planet: deforestation in Brazil. The research project of Tim Reutemann investigates how something as abstract as ,the prevention of deforestation“ is best paid for on the level of individual landowners. The experiment models farmers' land-use decisions under different payment contracts to evaluate which type of contract is most suitable.

Laboratory experiments usually involve high levels of abstraction and the visual presentation is kept as simple as possible. Interfaces are generally based on text and image output where necessary. Harrison and List [1] mention potential problems that arise from the framing in laboratory experiments and press for field studies in more 
natural environments as a solution. Using game design offers a middle course between the sterile laboratory and uncontrollable field settings. The Cattle Farming Game combines the key aesthetic features of simulation and farming games with the necessary functionality of economic experiments. It was tailored to the experiments of the ETH research project in Brazil, optimised for tablet computers and able to run on different devices using HTML 5, JavaScript and CSS. In essence, the Cattle Farming Game exemplifies the possibility of a Serious Economic Game, relying on an ,aesthetic“ approach to game design.

\section{Games and Immersion}

Digital games are the most recent addition to games, and enjoy huge popularity. The number of gamers - a term commonly used for players of digital games - is constantly growing worldwide. The very first computerised games appeared around 1950. The earliest examples were played on mainframes; for instance, OXO in $1952-$ an adaption of the paper and pencil game tic-tac-toe. They only reached a large audience with the invention of the personal computer.

Since then technology has changed considerably and more sophisticated graphics and improved controls as well as new ways of playing, have emerged. Today, digital games are played on personal computers, consoles and mobile devices such as smartphones and tablet computers. The increased popularity of digital gaming is accompanied by a shift away from analogue games [2]. Abt already hints at qualities that make digital games stand out: "Games offer expanded possibilities for action in a mode that, while chiefly mental, includes the felt freedom, intuitive speed, and reactive responses of physical movements" [3]. While present in any kind of game, this aspect of responsiveness, combined with expanded possibilities, is especially present in digital games.

\subsection{Immersion}

The ability to draw people in is a trait that traditional and digital games share. Often the term "immersion" is used to describe this effect. Games can provide a welcome distraction from everyday problems to the point where players forget the world around them. They generate a state of high concentration in which players feel as if they were "in the game" rather than in the real world. [4]

Immersion in games can also be described using Csikszentmihalyi's flow theory [5]. Flow occurs when the challenge and ability level of a task are well balanced. It is "the state in which individuals are so involved in an activity that nothing else seems to matter". Csikszentmihalyi mentions components of flow that are similar to immersion: high degree of concentration, a loss of the feeling of self-consciousness, distorted sense of time, direct and immediate feedback. [4]

Brown and Cairns isolated three levels of immersion: engagement, engrossment and total immersion [6]. The levels were defined from a study of gamers' experience. Using this distinction, a far more graded classification of immersive experiences is 
enabled. In contrast to the flow state, which is only rarely reached, immersion is something that does not need to be so extreme [4]. Total immersion is difficult to achieve because of both human and game limitations. Engagement describes the players' prior motivation to to engross himself in a game. If they do not like the idea of a game, they most probably will not even try to enjoy playing it. Access can also be prevented by the game controls or the time effort necessary to get into the game world. Once the obstacle of engagement is overcome, the player may get more involved to a level that Brown and Cairns call engrossment. Players have already invested a lot of time and effort and have therefore made an emotional connection to the game. They are less aware of their surroundings. It is described as "A Zen-like state where your hands just seem to know what to do, and your mind just carries on with the story" [6]. The amplification of that state leads to total immersion. Participants describe being cut off from reality and are fully focused on the game. Quotes from the study illustrate this: "When you stop thinking about the fact that you're playing a computer game and you're just in a computer." [6]

Graphics, plot and sound are mentioned to make up the atmosphere of the game. They contribute to immersion, when they demand attention. "The more attention and effort invested, the more immersed a gamer can feel" [6]. Immersion is therefore dependent on involvement with a game. While total immersion is a fleeting state, engagement and engrossment are more likely to occur.

Even though immersion depends on players' engagement, it is not exclusively under their control. Sometimes immersion in a game also leads to sub-optimal experiences. Jennet et al. remark that "people do not always play games because they want to get immersed, it is just something that happens. It does seem though from previous work that immersion is key to a good gaming experience" [4].

\section{Economic Experiments in the Form of a Game}

Economic experiments are a common tool for economists to test human behaviour in economic situations. The goal is to test general theories in respect to their predictive capability of actual behaviour. Charles Plott explains the logic as follows: "General theories must apply to simple special cases. The laboratory technology can be used to create simple (but real) economies. These simple economies can then be used to test and evaluate the predictive capability of the general theories when they are applied to the special cases." [7]

\subsection{External Validity}

The way experiments are framed in a laboratory influences how subjects behave. For instance, it is clear that the anonymity towards other players and the fact that one will never play against them again is a trait of laboratory experiments not found in real-life situations. Therefore, with all results gained from experiments in such a setting, the question of external validity is raised: What does the experiment say about the real world? According to Schmidt "external validity refers to the question [of] whether we 
are drawing the right conclusions from the experiment about the real world." [8] Testing theories is only one role that experiments play in economics. They can also serve as "material models" and reveal new phenomena that current models do not take into account and can therefore generate truly new insight.

\subsection{Economic Models}

Like all models, economic models point to the real world. They capture a system or specific aspects of it. While experiments face the problem of external validity, economic models often face the problem of realism: Do they capture all necessary aspects of a situation to provide an accurate prediction?

Most economic models since the 1950s have had a mathematical structure. Schmidt argues that models can also have a far less formal structure, as they can also be a physical object for instance. In this way, economic experiments can be viewed as material models. In the same way that engineers use plywood models of an airplane to predict how a real airplane behaves under different airstream conditions, economists have used the laboratory as a "wind tunnel" to test the design of real-world incentive mechanisms: "The experiments used simplified auction rules, students as subjects, and financial incentives that were many orders of magnitude smaller than the money at stake in the real auction. Nevertheless, the insights from the auctions in the lab were very useful to improve the design of the real auctions." [8]

\subsection{Serious Economic Games}

A recent acknowledgement of the importance games have in our lives has led to a new look at game design. A field called Serious Games has emerged. Their primary goal is not entertainment, but the appliance of game-design and -technology to achieve objectives outside the game setting. This does not mean that Serious Games are not entertaining. In fact, the exact opposite is the case, since the motivational and entertaining aspects of games are the qualities that Serious Games try to harness.

Games too are models in that they represent some aspects of a complex system in an abstracted way, which is easier to grasp. They can therefore represent not only fictional realities, but also the real world. Man has used games to experiment and experience beyond his own limitations, opening up new ways of learning and gaining insight into how the world works [3]. In this fashion a game can serve as an economic model. And thanks to its accessibility, it can be easily put to the test in an experiment.

The use of a game for an economic experiment is a very specific application of a Serious Game; one that, in order to be valid, has to meet various demands. Furthermore, it is a utilisation of a game that contrasts with a long tradition of economic experiments focused on simplicity and abstraction. An example of the reduced visual appearance of experiment interfaces can be seen in the Zurich Toolbox for Readymade Economic Experiments (z-Tree) [8]. The unnatural reduction may distort the results, as mentioned by Harrison and List, who propose field experiments under more naturalistic circumstances [1]. Virtual worlds or games could serve as an alternative to complicated field experiments. They can create a setting that is as close 
in perception as possible to the real world [9] and have the potential to provoke a naturalistic player response. Subjects will play the game in a situation similar to classical economic experiments. Alternatively, the experiment could be conducted online, with players playing the simulation on their own computers. This requires a technical solution to pay out financial incentives online, which is not yet developed. Playing online would have the added advantage of the benefits found in virtual world and Internet experiments, for instance bigger sample size [10].

\section{Game Aesthetics}

The current focus of game researchers lies on social and technological aspects. Meanwhile, aesthetics have taken a minor role so far. Some scholars even point at possible problems with a game design methodology, that focuses on visual representation questions, rather than mechanics, which they consider to be at the core of game design [11]. Fullerton for instance describes in the book Game Design Workshop [12] extensive guidelines on how to create a game with a "playcentric" approach. While elaborating in great detail on formal and structural elements of games, he notably neglects aesthetic aspects, mentioning them only once: "Aesthetics are the visual and aural dramatic elements of your game, which we have told you repeatedly not to worry about for your physical prototype". He further advises to only sparsely involve aesthetics in the digital prototype as well. While this approach enables a focus on mechanic aspects of games, it does not do justice to the aesthetic opportunities of game design. Niedenthal mentions examples such as "Weight Chess" and "Spice Chess" by artist Takako Saito or the music-shooter Rez in which aesthetic properties are an integral part of the game design [13]. He notes: "Many current definitions of games focus on games as systems of rules, and the key activity of the designer is to manipulate the mechanics of the game through 'tuning' and 'balancing.' These ways of thinking about games gain additional force from a certain essentialism that has come from ludology, in which the playful core elements of digital games are often explicated through the example of simpler games".

Applying a "playcentric" approach to game design for a Serious Economic Game results in a conflict with the economic requirements. Since the mechanics of the embedded model are necessarily bound to the real world, they do not allow game design in the sense of manipulation of this model. As a consequence, the representation of the virtual world model is the only mean to enable and enhance an immersive experience. An alternate perspective on game design is required in what could be called an "aesthetic" approach.

An "aesthetic" approach builds on the assumption that rules and game mechanics are not the only means of creating the game experience. Games like Journey by game designer Jenova Chen show opportunities of a game design centered around aesthetic qualities. Without classical narration the visuals convey the goal and give meaning to the game. The mysterious mountain in the distance gives the player a direction. Fascinating landscapes pull the player into the virtual world and make the experience 
worthwhile. Even though the mechanics of the game are simple and familiar, this aesthetic focus lead to the game's critical and commercial success.

Malone found that the visual representation of game elements plays an important role in creating the fun and enjoyment we have playing games [14]. He argues that a visual representation that has similarities to the real world elicits a fantasy of objects that are not physically there. These fantasies have two aspects that make them important for games; they are emotionally appealing as a result of their allowing players to fulfil needs, and by working as metaphors they make the system easier to understand. Malone explains: "By a system with fantasy, I mean a system that evokes mental images of physical objects or social situations that are not actually present. For example, the Breakout and Darts games evoke images of physical objects like balls, bricks, darts, and balloons; and the omnipresent computer Adventure game evokes images of caves, dwarves, birds, and so forth." [14]

Game aesthetics, according to Niedenthal, do not solely describe but are often reduced to the graphical representation of a game. Other aesthetic aspects include music, sound and the controller through which we relate ourselves with game worlds [13].

\section{Guidelines for an Aesthetic Approach}

While it may still be unclear, if and how aesthetic qualities of games give rise to immersion and fun, it seems hard to refute that commercial games employ them in an effective way. The following section aims to extract aesthetic features embedded in existing games and put them into a more comprehensible structure.

The case studies were gathered from two sources, the Apple App Store and Facebook, and limited to games from the construction and management category as this would be the appropriate category for the Cattle Farming Game. Among the ten most popular games on the Apple App Store are five games that fall into that category (as of June 2013). They are: Clash of Clans, Hay Day, The Simpsons ${ }^{\mathrm{TM}}$ : Tapped Out, Megapolis HD and Family Farm Seaside. These games were examined more closely, as well as Skyscraper City, a Facebook browser game aimed at mobile devices. The results are condensed into seven aesthetic features:

\subsection{Isometric Perspective}

The term "isometric" is a common term for game graphics that use a form of parallel projection to represent a three-dimensional game world in a two-dimensional image. Usually it describes a dimetric projection that looks very similar to an isometric perspective, rather than a true isometric perspective. All of the construction and management games in the top ten on the Apple App Store use dimetric game graphics. The bird's eye perspective gives the player the needed overview to manage a virtual economy efficiently and keep track of its state.

Our eyes or a photographic camera see the world in a perspective projection. The dimetric projection appears slightly distorted, with near objects having the same scale as distant ones. But it saves a lot of computational power, which is especially important when designing for mobile devices. 


\subsection{Consistent, Detailed Visual Style}

The case studies employ detailed graphic assets. All examples strive for an evident likeness to the appearance of real objects. Even The Simpsons ${ }^{\mathrm{TM}}$ : Tapped Out with its flat shading has a high realism insofar as it refers to the animated series. In that sense it is an almost identical representation of the fictional world it depicts. In games this analogy to reality not only helps the player to understand what the displayed game element depicts, but he is furthermore able to draw conclusions as to what its function is. Since the earliest days of computer games, there has always been a tendency towards representational games and only fewer abstract ones were produced [15]. One reason for this, according to Wolf, is that the diegetic world is easier to enter into if it resembles the real world.

The default interface for controls is very much reduced. Most of the screen space is available to display the game world. Usually, only in the corners are some buttons displayed. Animation helps make the world feel alive and creates immersion. The trees in Hay Day softly sway in the wind, the chickens walk around and pick corn. In some instances the animations also help the player to understand the game. In Skyscraper City graphics of the buildings are static, but animations are shown on top of the building when the user initiates an action such as demolishing a building.

\subsection{Musical Score and Sound Bound to Diegesis}

Sound is an important element of games. All games examined in the case studies use sound. Family Farm Seaside and Hay Day use music on an endless loop in the background. On top of the musical score, some nature sounds of birds chirping are played. Different sounds for interaction are played when buttons are clicked. In Hay Day animals emit a typical sound of their species when selected. In The Simpsons ${ }^{\mathrm{TM}}$ : Tapped Out sounds are used in an almost identical fashion, with a background score and sounds for interaction.

Ekman proposes a framework for distinguishing sounds based on their meaning and relation to the game world [16]. He classifies sounds based on two aspects: the sound itself (signal) and the meaning that the sound signifies or the event to which it refers to (referent). Both can either be diegetic or non-diegetic. Diegetic describes something that exists in the game narration, while non-diegetic parts of a game are outside its narration, e.g. belonging to the game interface. Diegetic sound has both source and meaning in the game narration. They are seldom used. Some sounds, e.g. feedback for button presses and the music, abandon any connection to diegesis and qualify as non-diegetic sounds.

Whenever possible, game sound creates a link to the game world. The so called masking sounds originate from in-game entities like the voices of the Simpsons characters, but they signal the clicking event of the player, which does not bear any meaning in the game world and is therefore non-diegetic. They mask the player notification of some event outside the game narration with a sound inside the diegesis. The connection of sounds to diegesis seems to be a strategy to heighten player immersion. Ambient sounds such as birds chirping, may contribute to the perceived liveliness of 
the virtual world. The linking of sounds to game events also creates importance for the player. He can improve his performance when he pays attention to auditory cues. This motivates him to pay close attention to game sounds. Referring back to Brown and Cairns [6], immersion is linked to the player's investment in the game. Having them pay close attention to sound in addition to visuals generates more investment and therefore should increase the feeling of immersion.

\subsection{Visual Feedback through Infographics and Text}

The studied games use infographic elements, including icons, charts and textual information, to report on the state of the world. The game menu is one example of a visual feedback element, which is used in all case studies. It is displayed in the periphery of the game, usually using iconographic buttons in the corners of the screen. It is very common to show inventory, such as money an other ressources as a number and player progression with a bar chart. Icons can also appear over the game world. In The Simpsons ${ }^{\mathrm{TM}}$ : Tapped Out icons are used for the menu buttons and also as overlays over the play-field. For instance a thumbs-up symbol or a dollar sign appears over buildings and characters when money and experience points can be gathered. Skyscraper City displays icons over buildings in order to inform the player of required actions. An orange triangle with a light bulb appearing over a house, for instance, signals the lack of electric power. These overlaid graphics usually distinguish themselves from the play field through animation or other means such as borders. Dialogue windows are placed over the play-field and used to notify the player of events or display larger menus, e.g. the list of available buildings to construct.

\subsection{Splash Screen and Introductory Videos}

Upon first launching Hay Day an opening introductory video is played. After that, the first steps in the game are explained through an interactive tutorial. It teaches the player how to plant crops, how to harvest them and how to add new buildings and animals to the farm. A straw man serves as the player's personal assistant and explains what he has to do next. Overlaid animations and arrows show where and how to interact. The use of spash or start screens is a common feature. It set's the tone of the game and is usually illustrated with an image that refers to it's narration. Videos and interactive tutorials lead the player into the game world and serve as an initial trigger to engage the viewer in a playful way.

\section{Applying Theory to Practice}

Figure 1 shows an early draft of the Cattle Farming Game, using a very abstracted black and white interface to present the virtual farm. All the essential information and interaction possibilities for the simulation are present. This visualisation strategy is closely related to the visual appearance of conventional economic experiments conducted with computers. 


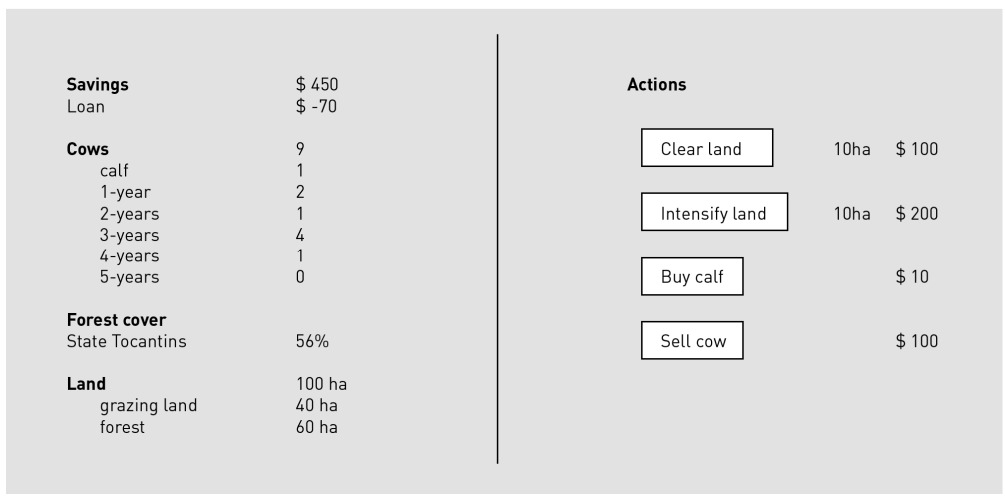

Fig. 1. Text-based draft of the Cattle Farming Game

Depending on the definition, this could already qualify as a game, given that all the mechanics of the economic model are in place. Yet to harness the full power of games, a more strict definition including aesthetic qualities is necessary. Accordingly, the aesthetic guidelines were applied to the Cattle Farming Game in an iterative design process. A detailed, dimetric visual representation of the virtual world was developed. The game world serves three distinct purposes in the Cattle Farming Game: It first and foremost shows the player information about the state of the virtual world. One can read the amount of forest left on the land and see where and how many cows belong to the farm. Also, players will notice when fertilisation wears off or the land degrades after too many cows have been grazing on it. A second and equally important function is interaction; buying cows or altering the land is done directly in the game world. This allows the player to decide not only to buy a cow, but also where to put it and similarly where to deforest, if he wishes to do so. The third function is immersion. Having a detailed representation enables the player to engage on an emotional level and demands his attention.

\subsection{Abstraction vs. Photorealism}

The analysed case studies employ aesthetics from a very abstracted, comic-like style as seen in The Simpsons ${ }^{\mathrm{TM}}$ : Tapped Out to high fidelity, almost photorealistic graphics more commonly found in modern first perspective games. In a study of a first-person-perspective Serious Game, realism was identified as an important motivational aspect. "Realism was the most commonly mentioned reason to continue playing the game. Some participants also mentioned that a lack of realism was a reason that they did not find parts of the game motivating" [17]. Another possible problem with abstraction in a Serious Economic Game has to do with cultural and individual differences. Pictographic depictions are similar to language as they rely on conventions. This effect can be minimised with a photorealistic approach. Subjects can easily relate to game images that resemble the real world as perceived through their eyes. Figure 2 shows the final game design. The photorealistic assets were created through detailed digital painting in the software Adobe Photoshop with a professional pen tablet as the 
primary input device. All drawings are based on photography of the targeted geographical region. The reference material was provided by Tim Reutemann of the ETH Zurich and collected during a research trip to the Brazilian state of Tocantins.

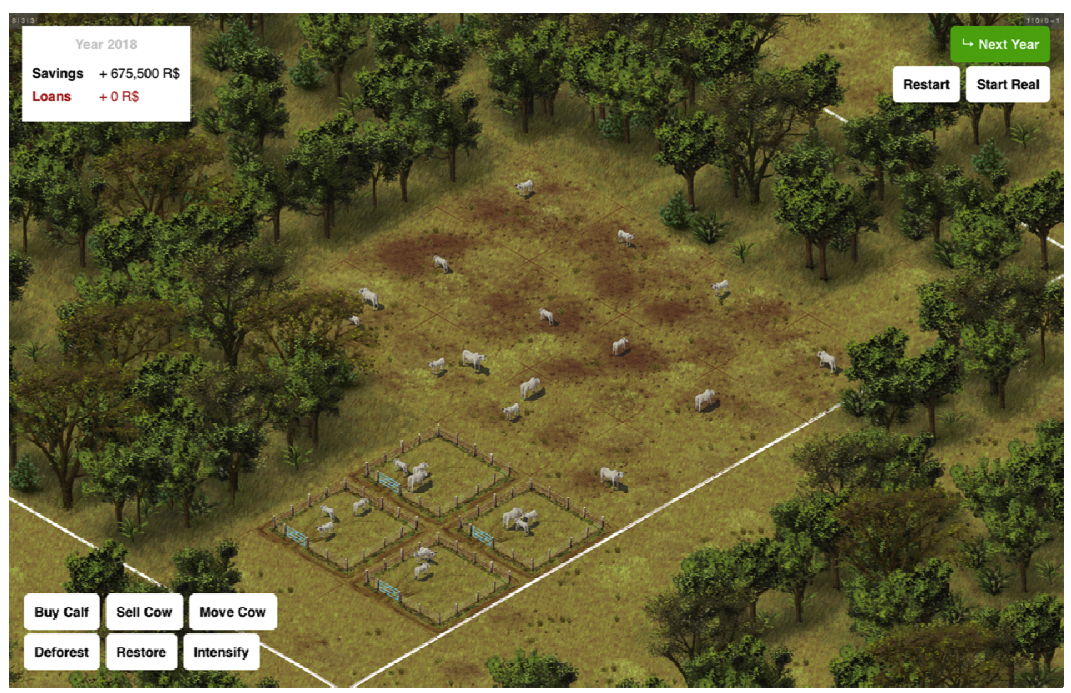

Fig. 2. Screen shot of the final Cattle Farming Game. In the process, a large number of improvements and changes to the graphics were made to enhance the look. Shadows under the trees and a grass texture with seamless transitions at tile borders, amongst other details, add to a harmonious image of the game world. All the game assets are drawn in the same near-photorealistic style.

The final visual representation of the farm closely resembles a real farm seen from an aerial perspective. Individual cows are visible in the field and each piece of land shows its condition. Hence, the player can gather information from the visuals. This makes the visual representation important for the user. As a consequence, players will feel more immersed than they would in front of a more abstract representation of the same information. Details such as randomly placed trees on forest patches conceal the repetitive grid-structure beneath the game world. Each forest tile is therefore unique and no repetitive pattern shown, which avoids breaking the illusion of the world.

\subsection{Sounds}

To create the right kind of atmosphere for the game, a relaxing, atmospheric and lowkey musical soundtrack was collected from Jamendo, a web platform with a large library of music published under Creative Commons licence. To further enhance the ambient sounds and complement the farming simulation, bird and nature sounds are laid over the music. The resulting soundtrack adds a deeper layer to the game and accommodates an even more game-like and enjoyable experience. Besides creating an atmospheric experience for gamers, sound also has the potential to make the game 
more accessible. Since accessibility is one of the important factors of an immersive game, players are informed of clicks with a sound feedback. This direct feedback heightens the perceived responsiveness. To achieve a connection to diegesis the sound effects relate to the meaning of the action in the game. For instance the restoring of a destroyed tile of land is underscored with a natural grass sound. The various sound effects were collected from freesound.org, a collaborative sound database.

The visual feedback of a grassy tile changing to a degraded tile is enhanced with a degradation sound playing simultaneously. The same applies to fertilisation wearing off, signalling the player's eventual need to fertilise or restore. The linking of sounds to in-game meaning and indirectly to visual cue, is a useful tool for the player since it helps to understand the significance of the event. He gains an advantage through listening to the sound, propelling immersion once again.

\subsection{Interaction}

Game controls enable the player to express himself in the game world. When they are designed well, they almost disappear and leave the player with a sense of control, as if he were interacting with the world itself rather than a device. Setting the Cattle Farming Game up to be played on a tablet computer proved to be very advantageous for its interaction. All inputs can be made with touches to the surface and players do not need to learn the use of the controller or keyboard. Apple, with the launch of the original iPad, and many companies after that, have established a widespread norm of tablet use. Today most people in developed countries know how to interact with touch-screens. Also, smartphones nowadays primarily rely on touch-screens, making it extremely intuitive for players to interact with a game on such devices.

\section{Conclusion}

This project proposes a design strategy for a Serious Economic Game. The ,aesthetic“ approach allows the creation of a detailed game world, while leaving the economic model of the experiment intact. The result - hopefully - is a game that engages the study participants and enables a naturalistic player response through immersion. The experiment of the ETH could already yield good preliminary results. The game design helped motivate players to participate. It managed to grab the attention of players for a long time and made lengthy test sessions possible.

Game aesthetics are an important part of game design, but are often left unanalyzed. If done correctly, an "aesthetic" approach can drastically change the success games have with their recipients. A game design strategy using all of the mediums strengths gets increasingly more important when real goals are at stake. Economic experimenting and Serious Economic Games are an ideal example, where this is the case. Yet the possibilities of such an aesthetic approach could reach beyond experimenting in economics and be adapted for various purposes of serious gaming. Generally it is a useful approach when designing a game relying on a rigid model. 
In the future, an aesthetic focus in game design could give a new point of view towards what makes games engaging. We still need to learn more about the role aesthetics takes on when we play games. A closer look and research that does not solely rely on case studies could define game aesthetics more precisely and therefore give a better understanding of these neglected aspects.

\section{References}

1. Harrison, G.W., List, J.A.: Field experiments. Journal of Economic Literature 42(4), 10091055 (2004)

2. Entertainment Software Association: Essential facts about the computer and Video game industry (2012), http: / /www. theesa.com/facts /pdfs/ESA_EF_2012.pdf

3. Abt, C.C.: Serious games. University Press of America (1987)

4. Jennett, C., Cox, A.L., Cairns, P., Dhoparee, S., Epps, A., Tijs, T., Walton, A.: Measuring and defining the experience of immersion in games. International Journal of Humancomputer Studies 66(9), 641-661 (2008)

5. Csikszentmihalyi, M.: Flow: The Psychology of Optimal Experience. HarperCollins, New York (1990)

6. Brown, E., Cairns, P.: A grounded investigation of game immersion. In: CHI 2004 Extended Abstracts on Human Factors in Computing Systems, pp. 1297-1300. ACM (April 2004)

7. Plott, C.R.: Will economics become an experimental science? Southern Economic Journal, 901-919 (1991)

8. Fischbacher, U.: z-Tree: Zurich Toolbox for Readymade Economic Experiments. Experimental economics 10(2), 171-178 (2007)

9. Haruvy, E.: Challenges and Opportunities in Economics Experiments in Virtual Worlds. Southern Economic Journal 78(1), 1-5 (2011)

10. Chesney, T., Chuah, S.H., Hoffmann, R.: Virtual world experimentation: An exploratory study. Journal of Economic Behavior \& Organization 72(1), 618-635 (2009)

11. Salen, K., Zimmerman, E.: Rules of Play. Game Design Fundementals. MIT Press, Cambridge (2004)

12. Fullerton, T.: Game design workshop: a playcentric approach to creating innovative games. Taylor \& Francis, US (2008)

13. Niedenthal, S.: What we talk about when we talk about game aesthetics. In: Barry, A., Helen, K., Tanya, K. (eds.) Breaking New Ground: Innovation in Games, Play, Practice and Theory. Proceedings of the 2009 Digital Games Research Association (2009)

14. Malone, T.W.: Heuristics for designing enjoyable user interfaces: Lessons from computer games. In: Proceedings of the 1982 Conference on Human Factors in Computing Systems, pp. 63-68. ACM (March 1982)

15. Wolf, M.J.: Abstraction in the video game. In: Wolff, M.J.P., Perron, B. (eds.) The Video Game Theory Reader, pp. 47-65. Routledge, New York (2003)

16. Ekman, I.: Meaningful noise: Understanding sound effects in computer games. In: Proc. Digital Arts and Cultures (2005)

17. Belanich, J., Orvis, K.L., Sibley, D.E.: Instructional Characteristics and Motivational Features of a PC-based Game. No. ARI-RR-1822. Army Research Inst for the Behavioral and Social Sciences Alexandria VA (2004) 